ITP-SB-79-86

Application of Catastrophe Theory to Nuclear Structure

by

G. Scharff-Goldhaber

Brookhaven National Laboratory, Upton, New York 11973

and

M. Dresden

Institute for Theoretical Physics

State University of New York at Stony Brook

Stony Brook, New York 11794

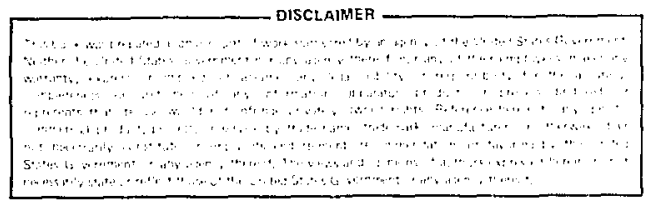

MASTER

Contributed to a Festschrift for Maurice Goldhaber

To be Published by the New York Academy of Sciences

The submitted manuscript has been authored under contract EY-76-C.0\%, 0016 with the U.S. Department of Energy. Accordingly, the U.S. Goverment. retains a nonexclusive, royalty-free license to publish or reproduce the published form of this contribution, or allow others to do so, for $U . S$. Government purposes. 
Application of Catastrophe Theory to Nuclear Structure by

\title{
G. Scharff-Goldhaber
}

Brookhaven National Laboratory, Upton, New York 11973

and

\author{
M. Dresden \\ Institute for Theoretical Physics \\ State University of New York at Stony Brook \\ Stony Brook, New York 11794
}

\begin{abstract}
Three two-parameter models, one describing an A-body system (the atomic nucleus) and two describing many-body systems (the van der Waals gas and the ferroelectric (perovskite) system) are compared within the framework of catastrophe theory. It is shown that each has a critical point (second order phase transition) when the two counteracting forces controlling it are in balance; further, each undergoes a first order phase transition when one of the forces vanisnes (the deforming force for the nucieus, the attractive force for the van der Waals gas, and the dielectric constant for the perovskite). Finally, when both parameters are kept constant, a kind of phase transition may occur at a "critical" angular momentum, "critical" pressure, and "critical" electric field.
\end{abstract}


It is a great pleasure to dedicate the following considerations to Maurice Goldhaber, whose elegant and Imaginative approach to physics is an inspiration and a source of constant pleasure to the whole physics community.

\section{1) Background}

It is obvious that nuclei are extremely complex systems consisting as they do of a finite but "large" number of protons and neutrons, interacting via strong forces which are only incompletely known. Even if it were known that all the relevant forces were two body forces, described by relatively simple interactions, the resulting many body problem would be so intractable that it would be difficult to draw a reliable conclusion from the necessarily approximate calculations. In addition, it is likely (as is the situation in solid state physics and statistical physics) that in different physical cir 'mstances different aspects of the many body problem dominate the physical description. Consequently, even if the possible importance of meson degrees of freedom, relativistic effects, and internal nucleon structure is ignored, the statement that a sufficiently detailed knowledge of the nucleon-nucleon interactions is sufficient to deduce the nuclear properties would be more in the nature of a scientific exhortation than of an implementable principle.

It is important to recall that in spite of the intrinsic complexity of nuclei, they exhibit a wealth of remarkable empirical regularities. It is in the best tradition of physics to first exploit these regularities by making a physical model whose physical and mathematical behavior 
simulates the nuclear properties in that particular donain. Later, the regularitles codifled by the model can be fit into a more general scheme, which starts from a more fundamental viewpoint. The origlnal shell model, or Individual particle model, exploits the many nuclear properties which can be described in terms of a few valence nucleons, while the collective model is in first instance based on the collective features of rotational spectra. The sheil model works well for nuclel near closed shells, whereas the collective description is most efficient away from magic number nuclet. In almost all of these models a certain amount of experimental information 18 used, as for example in obtaining the matrix elements of an effective Interaction.

In view of the complexity of atomic nuclei, it is clear that quite distinct approaches might be useful in obtaining new insights. One approach would be to deepen the understanding by investigating what concepts and rules of the models could be obtalned from a more detalled examination of the many body problem. For example, in the interacting Boson model (IBA), ${ }^{1}$ a specific microscoplc dynamical proposal ts made to account for the complex band structure exhibited in nuclear level schemes. In thia model certaln states of the "fermion" system consisting of an even number of both valence neutrons $\left(N_{n}\right)$ and protons $\left(N_{p}\right)$ is approximately descrijed in terms of an equivalent set of $N$ interacting Bosons $\left(N=\frac{N_{n}+N_{p}}{2}\right)$.

The other approach, which is dealt with in this paper, is to derive from the experimental regularities and the empirical rules "directly" a phenomenologlcal model, and to investigate the consequences and conclusions following from 1t. Once an effective model of some generality 
has been obtalned, one can attempt a fundamental justificacion of the concepts used. It often happens that the model suggested by the exper1ments ylelds new information via machematical analysis. In this connection it is Interest Ing to explore the mathematical organization which catastrophe theory ${ }^{2}$ Imposes on a general class of models. In Investgating the nature of the mathematical constralnts which follow $y$ la cataetrophe theory from the parameterized expertmental data, one follows the oppoattedirection of studying the return to a more wicroscople theory. In this approach the detailed nature of the interaction is not important, one fust investigates the possible frameworks in which the theory is required to fit by the present organization of the data. The particular class of phenomena to be explored here in a somewhat rentative fashion are the regularities observed in the exctied states of even-even nuclei.

2) The excited staces of even-even nucle1. The varlable moment of Inertia model.

Very striking emplrical regularities were observed in the ground state bands of even-even nuclet 1.e. bands with level spins (J) and parities $(\pi): 0+, 2+, 4+, \ldots$ In the introduction of a recent revlew article ${ }^{3}$ entitled "The Varlable Moment of Inertia Model and Theories of Nuclear Collective Motion," the sequence of discoverles underlying the model has been described, ranglng from rotational bands, near-harmonic bands (Interpreted by a surface vibration model, the first of the interacting boson models), bands founds in "transttional" (between vibrational and rotational) nuclel (in osmium and platinum), to bands found in near- 
magic and finally in magic nucled. While it was thought that all these bands in particular the first two, correspond to quite different modes of collective motions, the scaling law first observed by Mallmann ${ }^{4}$ suggested that all these bands result from one and the same mechanlam: Mallmann found that the ratio of energles $E_{J}$ (where $J=6$ or $B$ ) to the 2+ state excitation energy are universal functions of one ratio, $E_{4} / E_{2}$. One can therefore Infer that the ground state band spectra are functions depending on Just two parameters. 5 Mallmann also showed that the observed behavior devlates already very close to the rotational IImit from that proposed by Bohr and Mottelson, who expected a correction term for the energy $a(J(J+1))^{2}$ due to rotat lon-vibration mixing. Since an earlier, more fundamental approach, namely to find a general expresston for the energy spectrum of the form $E_{J}\left(\mathbb{N}_{p}, N_{n}\right)$ did not lead to success, the new challenge was to guess at the dynamics underlying the Mallmann curves, whose number could soon be extended from two to $61 x(J=16)$ or even higher thanks to new data. The two main avenues avallable were to postulate elther a rotational or a vbrational mechanism. If rotation is assumed, one has to conclude that Bince In general the energy spacings empirically found for Increasing $J$ are maller than those given by the expression for a rigld rotor, $\frac{\mathrm{J}(J+1)}{2 I}$, It follows that the moment of Inertia I Increases with Increasing J. Th1s increase can be accounted for by adding to the rotational term a term $\frac{C}{2}\left(I-I_{0}\right)^{2}$ (accounting for the Increase of centrifugal and Corfolis forces with increasing rotational velocity), 
together with a varlational principle required to determine I: The bastc equations for the Vartable Moment of Inert ia Model are then

$$
E_{J}(I)=\frac{1}{2} C\left(I-I_{0}\right)^{2}+\frac{J(J+1)}{2 I}
$$

$I_{0}$ and $C$ are parameters characteristic for a given nucleus. The moment of Inertla I Itself is a dynamical variable; tts dependence on the modeI parameters is given by

$$
\left.\frac{\partial E}{\partial I}\right|_{J}=0 \rightarrow I^{3}-I_{0} I^{2}=\frac{J(j+1)}{2 C}
$$

The second approach possible is the assumption that the dynamica underlying the Mallmann curves is that of the anharmonic vibrator. However, a comparison of the two approaches showed ${ }^{6}$ that the rotational interpretation is in considerably better agreement with the data. Becent experimental results for higher spin states in near-magic nuclei ${ }^{7}$ atrengthen this concluston. (It may be worth mertioning here that there is almost no correlation between the basic variable $E_{4} / E_{2}$ of the VMI model and the boson number $N$ up to $N$ i 11 .)

Finite positive values of $I_{0}$ and $C$ yield one real root for $I$ (which is determined from equation (2)). For $I_{0}>0, I_{0}$ can be interpreted as the ground state moment of inertia. One finds emplicically that $I_{0} r i s e s$ as more proton and neutron pairs are added to closed shells. C, the stiffness parameter, decreases by about 5 orders of magnitude between the nucleus ${ }^{12} \mathrm{C}$ and the heaviest actinides. Within a nuclear species, C rigeg as one approaches a stable 1sotope. 3 
It is most important that the model def Ined by equations (1) and (2) can be extended to negative values of $I_{0} ;{ }^{5,3}$ this extension was suggested by the observation that $I_{0}$ decreases sharply as magic numbers are approached and appears to become negatlve before $N=82$ (maglc number) is reached. One flnds good experimental agreement with (1) and (2) also for negative values of $I_{0}$. This means that while for $I_{0}>0$, $I(J-0)=I_{0}$, for $I_{0} \leq 0, I(J=0)=0,1, e$. the ground stase moment of tnertia vanishes and the nucleus actively resists being rotaced. This resistance is measured by the threshold energy $\frac{C}{2} I_{0}^{2}$. However, In contrast to Mallmann's findings (based on very scarce data), bands In magic nuclet $11 \mathrm{e}$ below the 1 imit of validity of the inodel $\left(E_{4} / E_{2}=1.82\right)$, for which $I_{0}=-\infty$. Below this $1 \mathrm{ln} 1 \mathrm{t}$, an abrupt change in band structure occurs; In particular, values for $4+, 6+, 8+$ etc. becone almost degenerate. As we shall see, this 1 imit corresponds to a first order phase transition. Moreover, it is easy to show that as $I_{0}$ changes sign, $\left.\frac{\partial I}{\partial J}\right|_{j=0}$ has a discontinuity reminiscent of a second order phase transition. We w11l show later that $I_{0}=0$ precisely corresponds to the critical point.

A phenomenon resembling a phase transition is further observed for the ground atate band of each ever-even nucleus at a state $J_{c}$, where $J_{c}$ denotes the "critlcal op $1 n^{\prime}$ : above $J_{c}$ a more or less abrupt deviaticn from the VMI prediction occurs, usually corresponding to a sudden increase in moment of inertia. For nuclel with many valence nucleon pairs $J_{c}$ is high (between 10 and $16 \mathrm{in}$ the rare earth region, $>20$ in the actinides.) For near-maglc nuclet" $J_{c}$ is usually $\leq 8$. For "pseudomagle nucle1," 1.e. nucle1 possessing $2(4)$ mroton holes and $2(4)$ neutrons, or 
vice versa, $J_{c} \leq 4$. However, in contrast to the large variation for $J_{c}$, the rotational velocity $\omega_{c}$ at which the deviation occurs, is approximately the same for all bands. ( $w$ is related to $I$ and $J$ by the semiclassical equation $I \omega=\sqrt{J(J+1)})$. Phenomenologically, this effect can be described by band crossing, caused by one of several possible physical mechanisms. In many cases this mechanism consists in the decoupling of a single neutron or proton pair in a high $j$ orbital under the Influence of the Corlolis force. Subsequent alignment of this pair along the axis of symmetry brings about a large contribution to the moment of inertia.

Before we proceed to the analysis of the VMI nodel, let: us briefly address the question of what can be learned from the UMI model about the organization of the rotating assembly of nucleons? Unfortunately the answer is tiat in addition to a number of previous efforts, a recent attempt ${ }^{8}$ to derive the model from a fundamental approach to collective motions of a nuclear many-body system has so far not succeeded, mainly because of the difficulties of taking the effects of the Paul princlple properly into account. However, it was possible to infer the configurations of the rotating assembly of nucleons by correlating the measured electric quadrupole transition moments with the moments of Inertia derived via VMI from the spectrum: ${ }^{9}$ for nuclel with not more than four valence nucleons of one kind (neutrons or protons), a rotating "alpha - dumbbell" model suggests itself, whereas for nuclel with $\geq 6$ valence neutrong and protons, a two-fluid model consisting of a superfluld and an inertial fluid gives excellent agreement with the data. 


\section{3) Ideas of catastrophe theory}

Thom and Mather proved a beautiful abstract mathematical classification theorem, which for the present purposes may be paraphrased in this way. Suppose one has a system described by $n$ variables, $x_{1} \ldots x_{n}$, and $s$ parameters $\xi_{1} \ldots \xi_{s}$. Suppose the physical states are given by

$$
\left(\frac{\partial v}{\partial x_{i}}\right)=0 \quad i=1 \ldots n \text {. }
$$

This is a set of points, of $s$ dimensions, call it $v_{s}$. To each point in $v_{s}$ corresponds a physical state. Now consider a projection of $\mathrm{V}_{\mathbf{s}}$ on the space $\xi_{1} \ldots \xi_{s}$. If the projection is one to orie, a knowledge of the $\xi^{\prime} s$ will define the physical state uniquely. If the projection is not one to one, a single set of $\xi^{\prime}$ 's will define a number of physical states. If one moves in the $\zeta$ space from a region where one state in $V_{s}$ corresponds to one set of $\xi^{\prime} s$, to a region where more than one state in $V_{s}$ corresponds to one $\xi$, one has gone from a ore phase region to a region of two or more phases. As the $E$ variables are changed In this manner, the system has undergone a discontinuous transition. Stated differently as one varies $\xi^{\prime} s$, the number and type of critical points (i.e., points where $\left(\frac{\partial V}{\partial x_{i}}\right)=0$ ), changes. The mathematical results referred to give an exhaustive classification of the changes in the number and type of critical points for certain values of $\mathrm{s}$. The result of Thom and Mather now asserts that near such a region in $V_{s}$, where these changes occur, one can obtain a canonical form for $V_{i}$, the effective potential. (Note this is only true near the boundaries). What the form is depends only on the number of $\xi$ 
varlables in question. For a 4 dimensional $\xi$ space there are 7 such basic forms. Consequently the potential can be written

$$
v_{s}\left(x_{1} \ldots x_{n}, \xi_{1} \ldots \xi_{s}\right) \cong v_{0}+\sum_{i=1}^{7} \alpha_{i} v_{i}(x, \xi)
$$

Here the $\alpha_{i}$ are constants, the $v_{i}$ are specific functions. In (4) $v_{0}$ is regular near the boundaries. The power of the theorem lies in the fact that the functions $v_{i}$ all of which satisfy $\frac{\partial v_{1}}{\partial x_{i}}=0$, have a universal form and can be determined once and for all. The Thom theorem determines these functions $V_{i}$ uniquely, up to a diffeomorphism which is a differentiable homeomorphism. (A homeomorphism is a one-to one mapping, which fs continuous and has a continuous inverse.) For differen numbers of parameters $\xi$ the functions $v_{i}$ have characteristically different forms. For $s=1,2$, the form of the corresponding potential is unique

$$
\begin{array}{lll}
s=1 & V=x^{3}+\xi_{1} x & \xi_{1} \\
s=2 & V=x^{4}+\frac{1}{2} \xi_{1} x^{2}+\xi_{2} x & \xi_{1}, \xi_{2}
\end{array}
$$

If one has three $\xi$ parameters, the effective potential can have a number of forms; one is

$$
s=3 \quad v=x^{5}+\frac{1}{3} \xi_{1} x^{3}+\frac{1}{2} \xi_{2} x^{2}+\xi_{3} x \quad \xi_{1}, \xi_{2}, \xi_{3} .
$$

There are two other possibilities for $s=3$

$$
v_{3}=x_{1}{ }^{2} x_{2} \pm x_{2}{ }^{3}+\xi_{1} x_{2}{ }^{2}+\xi_{2} x_{1}+\xi_{3} x_{2}
$$


The states are now given by

$$
\frac{\partial v_{3}}{\partial x_{1}}=0 \quad \text { and } \quad \frac{\partial V_{3}}{\partial x_{2}}=0
$$

These cases are called the swallow's tail, the elliptic and hyperbolic umbilic respectfvely.

For $s>3$ there are more complicated forms

for $V$. One of the forms for $s=4$ for the effective potential is

$$
V=\frac{1}{6} x^{5}+\frac{1}{4} \xi_{1} x^{4}+\frac{1}{3} \xi_{2} x^{3}+\frac{1}{2} \xi_{3} x^{2}+\xi_{4} x
$$

The states now must satisfy the equation $\frac{\partial V}{\partial x}=0$, which is a 5 th degree equation. It will be noted later that the best experimental fit for a VMI nodel with additional parameters allowed leads just to such 5 th degree equatlons

A glance at (5) shows that if a system is parametrized by two variables $\xi_{1}, \xi_{2}$, and the states of the system can be obtained as the minimum of some effective potential, this potential must be (locally)

$$
V=x^{4}+\frac{1}{2} \xi_{1} x^{2}+\xi_{2} x
$$

while the states must satisfy

$$
\frac{\partial V}{\partial X}=0 \Rightarrow x^{3}+\xi_{1} x+\xi_{2}=0
$$

Similarly if one wants to contemplate a description in which more than two parameters occur, one is compelled in this framework to go to $(5 c),(5 d)$, or (5e).

It is clear that depending on $\xi_{1}$ and $\xi_{2},(7)$ either has one or three 
real solutions for $x$. Thus corresponding to $\xi_{1} \xi_{2}$, there is either one state (one minimum of $V$ ) or two states $(+1$ unstable state) (two minima + one maximum.) The region in the $\xi_{1} \xi_{2}$ plane, where (7) has three real solutions for $x$, is separated from the region with one real solution by a cusp, whose equation is

$$
4 \xi_{1}^{3}+27 \xi_{2}^{2}=0 \text {, }
$$

(See figure 1)

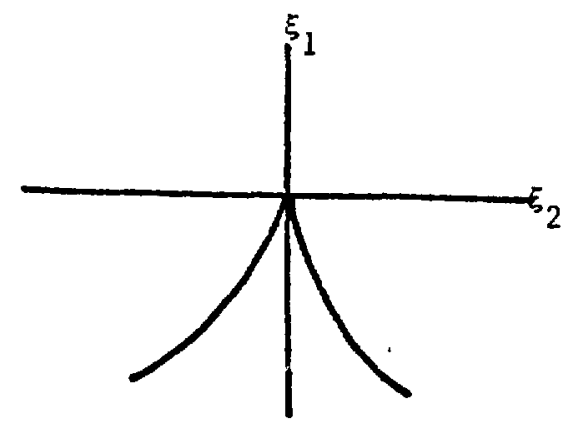

Figure 1

Eq. (B) can be obtained directly from the formula ior the discriminant of a cubic equation. In principle, crossing the cusp in the $\xi_{1} \xi_{2}$ plane corresponds to a discontinuous transition from a one-phase to a two-phase region. The Thom theorem is an abstract mathematical theorem. For its use in physics, the relation between the abstract parameters and physical quantities must be established. This must be done by casting the equations which characterize the physical states in one of the standard forms (5). In this process the relationship between the physical parameters and the mathematical $\xi$ parameters is not always so direct. It is presumed that such an identification is unique. In the cases treated here that is certainly true.

It is simple for the case of the van der Waals gas, where experiments give as equation of state 


$$
(\mathrm{p}=\text { pressure, } \mathrm{v}=\text { volume, } \mathrm{T} \text { absolute temperature }):
$$

$$
\left(p+\frac{a}{v^{2}}\right)\left(v^{*}-b\right)=R T
$$

( $R$ is the gas constant, $a$ and $b$, depend on the gas)

It is well known that there is a nitical point, with temperature, volume, pressure given ty

$$
\mathrm{p}_{\mathrm{c}}=\frac{1}{27} \frac{\mathrm{a}}{\mathrm{b}^{2}} \quad \mathrm{RT}_{\mathrm{c}}=\frac{8}{27} \frac{\mathrm{a}}{\mathrm{b}} \quad \mathrm{v}_{\mathrm{c}}=3 \mathrm{~b}
$$

For $T>T_{c}$ there is only a gas phase, for $T<T_{c}$ there are two possible phases. In that case, $\left(T<T_{c}\right)$ moving along a constant p line the system will undergo a gas liquid phase transition.

For $\mathrm{T}>\mathrm{T}_{\mathrm{C}}$ there is no phase transition. The van der Waals case fits exactly in the category (6) of Thon (1i). This can be seen explicitly by introducing new variables

$$
\pi=\frac{p}{p_{c}} \quad w=\frac{v}{v_{c}} \quad t=\frac{T}{T_{c}} \quad \text { in }(9),
$$

which leads to

$$
\left(\pi+\frac{3}{w^{2}}\right)\left(w-\frac{1}{3}\right)=8 t
$$

To recover a cinic of the form (6) write

$$
x^{\prime}=\frac{1}{w} \text {, and introduce } x=x^{\prime}-1
$$




$$
\begin{aligned}
& p^{\prime}=\pi-1=\frac{p-p_{c}}{p_{c}} \\
& t^{\prime}=t-1=\frac{T-T_{c}}{T_{c}}
\end{aligned}
$$

Substituting in (12) gives

$$
x^{3}+\frac{1}{3}\left(8 t^{\prime}+p^{\prime}\right) x+\frac{1}{3}\left(8 t^{\prime}-2 p^{\prime}\right)=0
$$

or

$$
\begin{aligned}
& x^{3}+\xi_{1} x+\xi_{2}=0 \\
& \xi_{1}=\frac{1}{3}\left(8 t^{\prime}+p^{\prime}\right) \\
& \xi_{2}=\frac{1}{3}\left(8 t^{\prime}-2 p^{\prime}\right)
\end{aligned}
$$

Thus the equation of state of the van der Waals gas liquid systen fits perfectly in the simplest cusp catastrope scheme of Thom. Observe that the critical point, eq. (10) in terms of $\xi_{1}, \xi_{2}, x$, is given by

$$
x=0 \quad \xi_{1}=\xi_{2}=0
$$

Note finally that since the van der Vaals equation is cubic in the density, the identification of $x$ with the density was forced by the equation defining the stable states (7). Also in the van der fials modeI, the potential $V$ itself has no especially transparent physical meaning. In the van der Waals case, the parameters " $\xi$ " of Thom are therefore by (16) and (17) 1inear combinations of pressure and temperature. Note finally that crossing the cusp 
produces a first order phase transition. More precisely: crossing the cusp, together with the Maxwell rule, yields a first order phase transition. (The Maxwell rule asserts that the stable isotherm, $\mathrm{p}=$ constant, bisects the area of the van der Waals loop.) At the critical point $\left(\frac{\partial p}{\partial y}\right)$ and $\left(\frac{\partial^{2} p}{\partial^{v}}\right)$ both vanish. This fixes $\mathrm{p}_{\mathrm{c}}, \mathrm{v}_{\mathrm{c}}$ and $\mathrm{T}_{\mathrm{c}}$.

4) Catastrophe interpretation of the variable mosent of inertia model

It is clear that the Thom classification can be directly applied to the VMI equations (1) and (2). The states are defined via a minimum condition on the energy by (2). The two parameters $I_{0}$ and $C$ determine the effective moment of inertia by the equation of state. This equation is again a cubic equation, so it can be put in the standard form (7). If one introduces in the equation (2) as a new state variable

$$
x=\left(I-\frac{1}{3} I_{0}\right)
$$

one finds that the equation which determines this

"difference moment of inertia" is again of the form

$$
\begin{aligned}
& x^{3}+\xi_{1} x+\xi_{2}=0 \quad \text { with } \\
& \xi_{1}=-\frac{1}{3} I_{0}^{2} \\
& \xi_{2}=-\left(\frac{2}{27} I_{0}^{3}+\frac{J(J+1)}{2 C}\right)
\end{aligned}
$$


This formal identity implies that in principle all the phenomena occurring in the van der Waals gas have their counterpart in the varlable moment of inertia model. There is a critical point given by $x=0, \xi_{1}=\xi_{2}=0$, $I_{0}=0, \quad J=0$.

The remark made earlier that $\left(\frac{\partial I}{\partial J}\right)$ at $\mathrm{J}=0$ has $a$ discontinuity for $I_{0}=0$ and corresponds to a phase transition of the second kind, is in precise harmony. with the observation that $I_{0}=0, J=0$, corresponds to the critical point. It is further interesting to observe that the cusp given by equation (8) can by the identifications (21) and (22) be transcribed to the form

$$
I_{0}^{3}=-\frac{27}{8} \frac{J(J+1)}{C}
$$

This very simple relation shows the formal analogy between the van der Waals description of a gas and the Variable Moment of Inertia description of a nucleus. Both fit in the cusp catastruphe scheme of Thom, both can be expected to exhibit phase transition type behavior. Although there is formal identity between the two descriptions, there are, as will be shown below, characteristic physical di_ferences which preclude a direct identification of the phenomena.

5) Discussion and Interpretation

a) It is easy to see by inspection that in the van der Waals case $\xi_{1}$ and $\xi_{2}$ can both be positive and negative. Any straight line in the $\xi_{1} \quad \xi_{2}$ plane in the van der Waals case can represent a sequence of physically possible temperatures and pressures. For example the line $\xi_{1}-\xi_{2}=$ constant represents a constant pressure line. In general, lines could intersect the cusp twice, each intersection (except at $\xi_{1}=\xi_{2}=0$ ) corresponding to a first 
order phase transition. The situation is different for the VMI model; because of (21) $\xi_{1}$ can only assume negative values, so only the lower part of Figure 2 has a physical significance. One can deduce from the basic formulae that for given $I_{0}>0$, a sequence of different spin states (varying $J$ ) are given hy a straight line parallel to the $\xi_{2}$ axis which ends at the cusp. That state is the $J=0$ state.

Figure 2

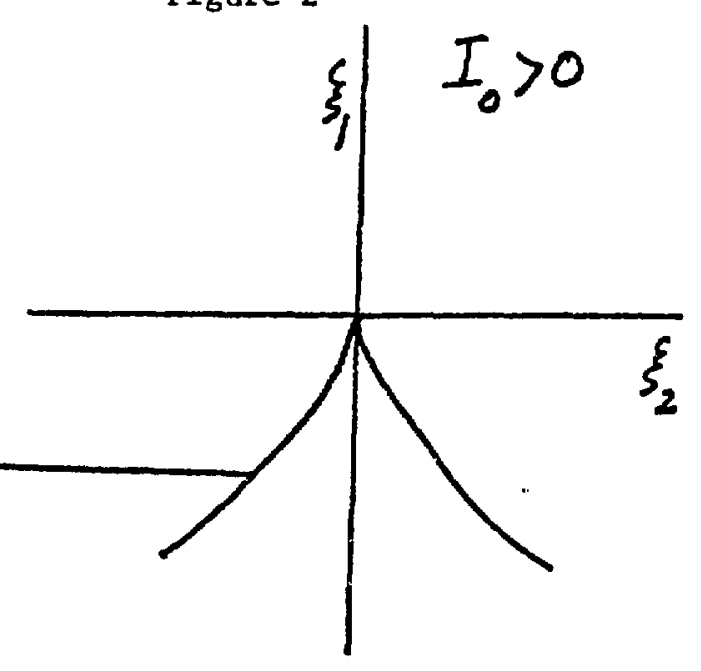

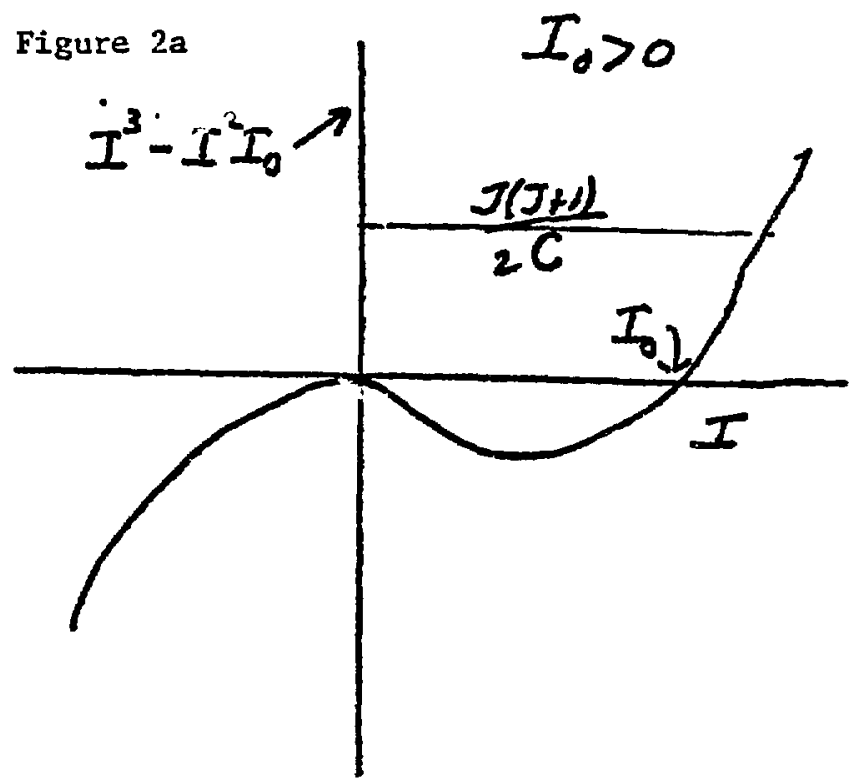

In that case (for $I_{0}>0$ ) there is for each $I_{0}, C, I$, only one stable moment of inertia $I$ of the nucleus (Figure 2). For higher spins $\xi_{2}$ decreases monotonically. As can be seen from (2) the baslc relation between $I, I_{0}, J$ and $C$ may be written as

$$
I^{3}-I^{2} I_{0}=\frac{J(J+1)}{2 C}
$$

In figure (2a) we have graphed $I^{3}-I^{2} I_{0}$ for $I_{0}>0$. since $\frac{J^{\prime}(J+1)}{2 C}$ is always positive, equation (2) (as is evident from Figure 2a) for given 
$I_{0}$ has always one positive solution for $I$.

On the other hand, if $I_{0}<0$ (FIgure 3 ), the sequence of nuclear states for different $J$ values starts at the $\left(\xi_{2}\right)$ positive branch of the cusp for $J=0$, and proceeds for larger $J$ to negative values

Figure 3

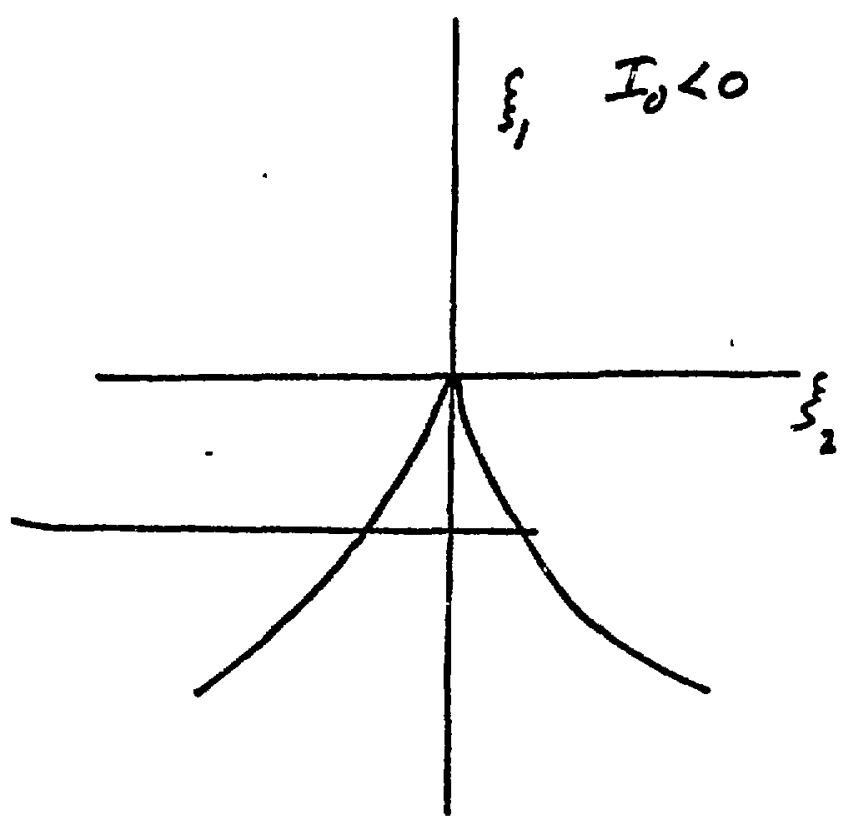

Figure 3a

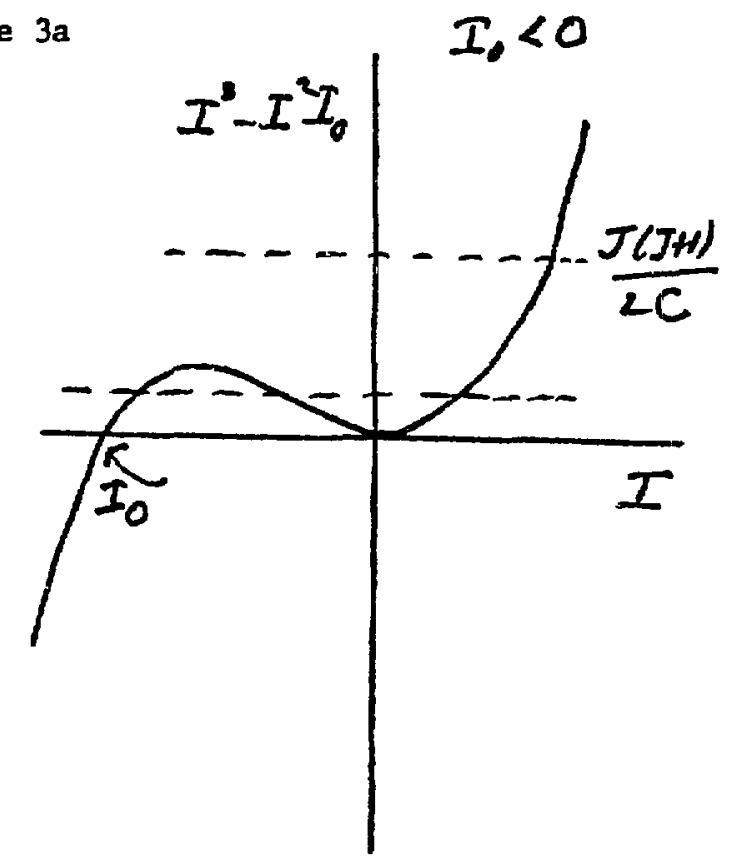

Then for negative $I_{0}$, there exists a sequence of nuclear states where $I$ can have three real values. If one again graphs $I^{3}-I^{2} I_{0}$, but row for negative $I_{0}$, one obtains Figure 3a. It is now Indeed clear (Erom the figure) that for a range of spin values there are three real solutions for $I$, for given $I_{0}$ and $C$. However only one of these has a positive moment of inertia $I$, so only one corresponds to a plisical state. Furthermore, as $J$ increases fron the "3 root region" to the one root region, the positive root changes continuously. Consequently, when in Figure (3) one passes the cusp, there 
will not be a discontinuous change in $I$; hence no phase transitiou type phenomenon occurs, in contrást to the van der Waals case.

b) There are other physical situations exhibiting a similar phase transition type behavior. A very instructive example is that of ferroelectricity. For certain substances called perovskites there can, at temperatures $T<T_{C}$, exist a spontaneous polarization, while for $T>T_{C}$, no such polarization exists; such a substance is $\mathrm{BaTiO}_{3}$. In all three cases the physical phenomena result from the competition between two opposing tendencies. In the van der Waals case these tendencies are just the repulsive and attractive regions of the intermolecular forces (characterized schematically by the $a$ and $b$ terms respectively). The pressure plays the role of an external influence on the system. It is therefore not surprising that the pressure is related to the Thom $\xi$ parameters. In the Variable Moment of Inertia model, the competition refers to the tendency to preserve the spherical shape of the closed shell which conflicts with the tendency to deform the nuclear shape. The Pauli principle tends to make the nucleus spherical, while the residual forces between valence nucleons, usually taken to be pairing forces and quadrupole quadrupole interactions, cause deformation. The rotation of the nucleus, via the Coriolis force, brings about a decrease of the pairing interactions. The parameter $C$ reflects the stability of the nucleus in question (i.e. the balance of the Coulomb repulsion and the symetry term) as well as the degree of surface rigidity which is related to shell filling. Io decreases from a maximum value at the center of a given proton and neutron shell to zero at about two nucleon pairs away from magic. It reaches $(-\infty)$ at the magic numbers. In the ferroelectric case these tendencies 
refer to the polarization $P$, which tends to pull the crystal apart and the elastic forces tending to restore the original shape. An external electric field E plays a role similar to the rotation. The basic formulae describing a ferroelectric crystal are $\mathrm{e}^{12}$

$$
\mathrm{P}^{3}+\gamma\left(\mathrm{T}-\mathrm{T}_{0}\right) \mathrm{P}-\alpha E=0
$$

$E=$ external field,$\quad P=$ polarization, $\alpha=$ constant

$Y$ is a constant, $T$ the temperature, $T_{0}$ a constant.

The term $\gamma\left(T-T_{0}\right)$ is directly related to the dielectric constant $\varepsilon_{0}$ of the material. A very good fit is obtained with

$$
\epsilon_{0}=\frac{a}{\gamma\left(T-T_{0}\right)}
$$

When $\epsilon_{0}$ passes through infinity, the linear term in (24) changes sign. This signals a phase transition (of the second order if $E=0$, of the first order if $E \neq 0$ ). The three physically sidilar cases correspond to the same Thom category. It is obvious that (24) is already in the standard form $x^{3}+\xi_{1} x+\xi_{2}=0$. It is equally obvious that $x$ has to be identified with the polarization. In Table $I$, the parameters $\xi_{1}, \xi_{2}$, and the variables $\mathbf{x}$ are collected for the three cases.

\begin{tabular}{c|c|c|c} 
& van der Waals & ferroelectric & VMI \\
\hline$x$ & $\frac{v_{c}-v}{v}=\frac{\rho}{\rho_{c}}-1$ & $P$ & $I-\frac{1}{3} I_{0}$ \\
$5_{1}$ & $\frac{1}{3}\left(8 t^{\prime}+p^{\prime}\right)$ & $\gamma\left(T-T_{0}\right)=\frac{a}{\epsilon_{0}}$ & $-\frac{1}{3} I_{0}^{2}$ \\
$5_{2}$ & $\frac{1}{3}\left(8 t^{\prime}-2 p^{\prime}\right)$ & $-\alpha E$ & $-\left(\frac{2}{27} I_{0}^{3}+\frac{J(J+1)}{2 C}\right)$
\end{tabular}


What do the correspondences shown in Table I mean in physical terms? A glance at Tæble I shows that $\frac{v_{c}-v}{v}=\frac{\rho}{\rho_{c}}-1 \quad$ ( $\rho$ is the density) p lays the same role as does $I-\frac{1}{3} I_{0^{\circ}}$. That is in harmony with an earlier suggestion $^{13}$ which relates I to the densi- $\mathrm{V}$. The suggestion ${ }^{13}$ that $\mathrm{J}$ corresponds to the pressure and $I_{0}$ to the temperature, is essentially confirmed, but needs some modification. From Table $\mathrm{I}$ one sees that $\mathrm{I}_{0}$ and $\mathrm{J}$ are determined by both pressure and temperature ( $t$ ' and $p^{\prime}$ ).

$$
I_{0}\left\langle>\sqrt{-\left(8 t^{\top}+p^{\prime}\right)} ; \quad \frac{J(J+1)}{2 C} \Leftrightarrow-\frac{1}{3}\left(8 t^{\prime}-2 p^{\prime}\right)-\frac{2}{27}\left(\sqrt{-\left(8 t^{\prime}+p^{\prime}\right)}\right)^{3}\right.
$$

Similarly, from Table $I$ it is seen that the parameter $I_{0}$ is related to the dielectric constant and the spin $J$ to tho electric field E.

These three cases, all belonging to the same Thom category exhibit similar phase transition behavior. As $I_{0} \rightarrow-\infty$, the VMI model undergoes a first order phase transition. Physically, as $I_{0} \rightarrow-\infty$, the nucleus cannot be cranked, an external torque will not produce rotation. In order to produce rotation one or more nucleon pairs must be promoted to a higher state, thereby deforming the nucleus. This deformed nucleus can then be rotated. A very similar behavior occurs in the ferroelectric case. As $\epsilon_{0} \rightarrow 0$, no externally imposed field can produce an internal electric field in the solid. In the van der Waals case, if $a=0$, i.e. there is no at:tractive force, the critical temperature $T_{c}=0$. This implies that it would take infinite pressure to liquefy the gas.

Let us now consider the phenomenon referred to earlier, namely the deviation of nuclear ground state bands from the VMI predictions at critical spin values $J_{c}$ ("critical" is used here in a sense which is related, but 
not identical to the use in "critical" phenomena), so that for spins larger than $\mathrm{J}_{\mathrm{c}}$ the moment of inertia increases dramatically. The comparisons given earlier allow an understanding of this phenomenon in terms of the ferroelectric mode1.

In the nuclear case the physical interpretation is that once the nucleus rotates so fast that the centrifugal and Coriolis forces overbalance the pairing forces, the moment of inertia will approach that of a rigid rotor. Similarly, in the ferroelectric case, once $E$ becomes larger than the elastic forces, further increase in $E$ does not yield a substantial increase in polarization. In the comparison with the van der Waals case $\mathrm{J}$ corresponds to $\mathrm{p}^{\prime}$, implying a phase transition from gaseous to liquid.

It is of interest to note here that for the heaviest nuclei no backbending was observed up to high spin states. Indeed, for ${ }^{238} \mathrm{U}$ the ground state band energies ${ }^{15}$ can be fitted ${ }^{16}$ up to $\mathrm{J}=28$ (highest level known so far) within $\lesssim 1 \%$ by the two-parameter VMI model, i.e., $\mathrm{J}_{\mathrm{c}}>28$. An even much better fit is achieved by an expression of the form ${ }^{6}, 14$

$$
E_{J}(I)=\frac{1}{2} c\left(I-I_{0}\right)^{2}+\frac{J(J+1)}{2 I}+\alpha\left(I-I_{0}\right)^{3}+\beta\left(I-I_{0}\right)^{4}
$$

fifth and sixth order expressions for $E_{J}$ (I) do not improve the quality of the fit. Together with $\frac{\partial E}{\partial I}=0, E q$. (27) yields a fifth degree equation for $I$, corresponding to the Thom category $5(e)$ which has as special cases all the qualitative features mentioned for the equations containing fewer parameters, but there are added refinements. Thus from a systematic and empirical viewpoint, this expression appears to be preferred.

The various correspondences one can derive from Table I essentially confirm those arrived at earlier ${ }^{13}$ on more intuitive grounds. However 
the analysis presented here provides a substantially deeper insight in +'e subtle relationship between variables and parameters of these three sys cems.

ACKNOWLEDGMENTS

This work was supported by the U.S. Department of Energy, Contract 非EY-76-C002-0016, and NSF Contract 非PHY 79-06376. 


\section{References}

1. F. Iachello and A. Arima, Phys. Lett. 53B, 309 (1974);

A. Arima and F. Tachello, Ann. Phys. N. Y., 99, 253 (1976);

J. B. McGrory, Phys. Rev. Lett. 41,533 (1978).

2. R. Thom: Structural Stability and Morphogenesis, W. A. Benjamin (1975);

M. Dresden: Fundamental In'eractions in Physics, Plenum (1970).

3. G. Scharff-Goldhaber, C. Dover, and A. Goodman, Ann. Rev. Nuc1. Sci. (1976) and references therein.

4. C. Mallmann, Phys. Rev, Lett. 2, 507 (1959).

5. A. S. Goldhaber and G. S. Goldhaber, Phys. Rev. C 17, 1171 (1978).

6. T. K. Das, R. M. Dreizler, and A. Klein, Phys, Rev。 C 2, 632 (197C)

7. G. Scharff-Goldhaber, J. Phys. G: Nuc1. Phys. No. 11 (1979); Corrigenda, No. 2 (1980).

8. B. Buck, L, C. Biedenharn and R. Y. Cusson, Nuc1. Phys. A317, 205 (1979).

9. A. S. Goldhaber and G. Scharff-Goldhaber, Phys. Rev. C 17, 1171 (1978).

10. T. Poston and I. N. Stewart, Taylor Expansion and Catastrophe's, Pitman, London (1976).

11. D. Fowler, "Toward a Theoretical Biology," 26, 239, 317, Edinburgh University Press (1972).

12. C. Kittel: Introduction to Solid State Physics, John Wiley \& Sons, 1977.

13. G. Scharff-Goldhaber, 4th Int. Conference on Atomic Masses and Fundamental Constants, September 6, 1971, p. 272, Teddington.

14. Saethre et a1., Nucl. Phys. A207, 486-512 (1973).

15. E. Grosse et a1., Phys. Rev. Lett. 35, 565 (1975);

E. Grosse, priv. comm. to L. Peker.

16. L. Peker and S. Pearlstein, priv. comm. 